\title{
A Frequency-Domain Near-Field-to-Far-Field Transform for Planar Layered Media
}

\author{
İlker R. Çapoğlu, Member, IEEE, Allen Taflove, Fellow, IEEE, and Vadim Backman
}

\begin{abstract}
We report a frequency-domain near-field-to-far-field transform (NFFFT) for the numerical modeling of radiation and scattering in planar multilayered spaces. Although the results are equivalent to those of Demarest et al. (1996), the formulation is more compact, more stable, and applicable to observation angles in the lower half space. Furthermore, the results are presented in a vector-potential formalism that is more easily adaptable to existing free-space implementations. The NFFFT algorithm can be used in any differential-equation-based finite numerical method, including but not limited to the finite-difference time-domain (FDTD) method and the finite-element method (FEM).
\end{abstract}

Index Terms-FDTD methods, finite difference methods, finite element methods, near-field far-field transformation.

\section{INTRODUCTION}

$\mathbf{N}$ EAR-FIELD-TO-FAR-FIELD transforms (NFFFTs) involve the formulation of the radiated far field (or Fraunhofer field) in terms of near fields obtained via some finite numerical method. A free-space frequency-domain near-field-tofar-field transform (NFFFT) was developed for the finite-difference time-domain (FDTD) method by Umashankar and Taflove [1]-[3]. This NFFFT algorithm was later generalized to multilayered spaces in [4]. In [5] and [6], a direct time-domain NFFFT algorithm was introduced for the FDTD analysis of three-layered media using a transmission-line (TL) analogy. In this paper, we describe the frequency domain generalization and extension of this TL-based approach to general (possibly lossy) layered media. The resulting NFFFT algorithm can be used in conjunction with any finite-difference numerical scheme, including the FDTD and finite-element (FEM) methods. The results are equivalent to those in [4], with the following additions and improvements:

(a) The results are bounded and numerically stable for arbitrarily-large electrical conductivities within the layering structure. This is achieved by putting the recursive relations in a coordinate-independent form that is free of exponentially-increasing terms.

\footnotetext{
Manuscript received April 28, 2011; revised August 17, 2011; accepted September 14, 2011. Date of publication January 31, 2012; date of current version April 06, 2012. This work was supported by the NIH grants R01EB003682 and R01CA128641.

İ. R. Çapoğlu and V. Backman are with the Biomedical Engineering Department, Northwestern University, Evanston, IL 60208 USA (e-mail: capoglu@ieee.org; v-backman@northwestern.edu).

A. Taflove is with the Electrical Engineering and Computer Science Department, Northwestern University, Evanston, IL 60208 USA (e-mail: taflove@eecs.northwestern.edu).

Digital Object Identifier 10.1109/TAP.2012.2186253
}

(b) The far field is expressed in a uniform manner that is valid for an arbitrary observation angle, whether it falls within the upper or lower half space.

(c) A clear connection is maintained with the traditional vector-potential integral formulation. This helps the generalization of existing free-space NFFFT implementations which are usually formulated in terms of vector-potentials. The software implementer does not need to change the formulation of the radiated field based on the vector potentials $\boldsymbol{A}, \boldsymbol{F}$ [see (3)-(4)], which is common in existing free-space NFFFTs. The only component of the software that needs to be updated is the one that calculates the vector potentials $\boldsymbol{A}, \boldsymbol{F}$ using the scalar transmission-line Green's functions pertinent to the specific layering structure. The NFFFT is thus split into two independent modules, rendering it more amenable to modularization and code reuse.

(d) Explicit formulas are given for up to three layers in closed form. From a software-implementation perspective, a closed-form solution for a restricted geometry is a convenient starting point before attempting a full generalization to the most complete and complex solution. After implementing and validating the simpler three-layer NFFFT, the implementer is equipped with considerable experience for a more general NFFFT implementation. In the debugging stage, a lot of errors in the general NFFFT can be caught by checking it against the (already tested) three-layer NFFFT in a three-layered geometry.

The rest of the paper is organized as follows. In Section II, the theoretical formulation of the NFFFT is explained. A recursive algorithm is described for general layered media, and explicit formulas are noted for the special case of three or fewer layers. In Section III, a validation study is presented to confirm the accuracy of the method. In Section IV, an FDTD software package featuring the NFFFT in this paper is briefly introduced. Section V concludes the paper with a summary.

\section{THEORY}

The geometry of the problem is shown in Fig. 1 [7]. The scattering or radiating structure $A$ in Fig. 1(a) is embedded in a planar $N$-layered medium, with relative permittivities $\epsilon_{r_{0}} \ldots \epsilon_{r_{N-1}}$, relative permeabilities $\mu_{r_{0}} \ldots \mu_{r_{N-1}}$, and electrical conductivities $\sigma_{0} \ldots \sigma_{N-1}$ from top to bottom. For the phasor analysis to be valid, the materials that make up the structure $A$ and the layer materials have to be linear. The half space (upper or lower) containing the direction of observation 


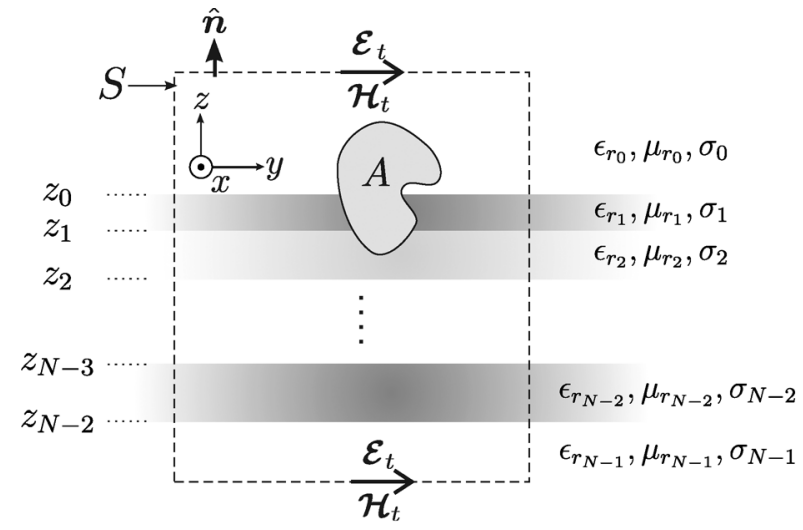

(a)

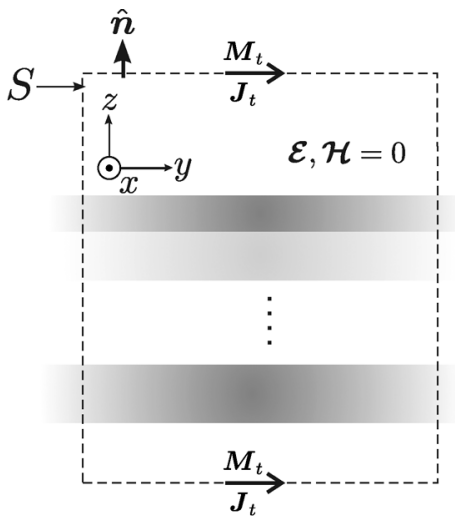

(b)

Fig. 1. Cross section of the radiation/scattering geometry in a planar layered medium. (a) The original geometry. (b) The equivalent geometry with surface currents.

is assumed to be lossless. In other words, if the observation direction is in the upper half space, $\sigma_{0}=0$; otherwise, $\sigma_{N-1}=0$. The $z$ axis of the Cartesian coordinates is aligned with the direction of stratification, and the $z$ coordinates of the $N-1$ layer interfaces from top to bottom are denoted as $z_{0}, z_{1}, \ldots z_{N-2}$.

A separate formulation for a NFFFT is rarely necessary for integral-equation-based numerical method such as the method of moments (MoM) because the Green's functions pertaining to the layered medium are already available. We therefore assume that a differential-equation-based finite method is employed, and the structure $A$ within the solution space is enclosed by a closed NFFFT surface $S$ as shown in Fig. 1(a). The dimensions and positioning of the NFFFT surface are arbitrary, as long as the surface contains the structure $A$. If the finite solution method operates directly in time-domain (e.g., FDTD), the time-dependent tangential electric and magnetic field components $\mathcal{E}_{t}(t)$, $\boldsymbol{\mathcal { H }}_{t}(t)$ on $S$ are converted to phasor values $\boldsymbol{E}_{t}, \boldsymbol{H}_{t}$ at frequency $\omega$ using on-the-fly discrete Fourier transform [3], [8]:

$$
\left\{\begin{array}{l}
\boldsymbol{E}_{t} \\
\boldsymbol{H}_{t}
\end{array}\right\}=\frac{1}{2 \pi} \sum_{n=0}^{N-1}\left\{\begin{array}{c}
\mathcal{E}_{t}(t) \\
\mathcal{H}_{t}(t)
\end{array}\right\} e^{-j \omega n \Delta t} \Delta t
$$

where $\Delta t$ is the time step and $N$ is the number of FDTD iterations. The true weight of the desired frequency component in the frequency spectrum of the waveform is obtained by applying the extra $1 / 2 \pi$ prefactor to the summation representing the Fourier transform. The sign of the complex exponent in (1) follows from the electrical engineering convention $\exp (j \omega t)$ for the harmonic time dependence of every field and source variable. Using the surface-equivalence principle [9], these tangential phasor field components are replaced by equivalent surface currents $\boldsymbol{M}_{t}, \boldsymbol{J}_{t}$ radiating in the layered space without $A$ as shown in Fig. 1(b). The equivalent surface currents are given by

$$
\boldsymbol{M}_{t}=\boldsymbol{E}_{t} \times \hat{\boldsymbol{n}}, \quad \boldsymbol{J}_{t}=\hat{\boldsymbol{n}} \times \boldsymbol{H}_{t}
$$

in which $\hat{\boldsymbol{n}}$ is the outward normal shown in Fig. 1(a). If the solution method operates directly in frequency-domain (e.g., FEM), then the equivalent surface currents $\boldsymbol{M}_{t}, \boldsymbol{J}_{t}$ are already available in phasor form.

\section{A. Dyadic Green's Functions for the Vector Potentials}

Let the spherical coordinates $(r, \theta, \phi)$ be centered around the origin, and the angle variables $\theta$ and $\phi$ denote the longitudinal angle with respect to $z$ and the azimuthal angle in the $x y$ plane, respectively. The $\theta$ and $\phi$ components of radiated electric field at the observation direction specified by the angles $(\theta, \phi)$ can be directly obtained from the vector potentials $\boldsymbol{A}, \boldsymbol{F}$ as follows [9]:

$$
\begin{aligned}
& E_{\theta}^{r}=-j \omega A_{\theta}-j \omega \eta^{o} F_{\phi} \\
& E_{\phi}^{r}=-j \omega A_{\phi}+j \omega \eta^{o} F_{\theta},
\end{aligned}
$$

in which $\eta^{o}=\sqrt{\left(\mu_{r}^{o} \mu_{0}\right) /\left(\epsilon_{r}^{o} \epsilon_{0}\right)}$ is the wave impedance of the observation half space (index 0 or $N-1$ ), and $\epsilon_{r}^{o}, \mu_{r}^{o}$ are the relative permittivity and permeability in the same half space, respectively. In the radiated-field zone, the vector potentials can be written as

$$
\begin{aligned}
& \boldsymbol{A}=\frac{\mu_{r}^{o} \mu_{0}}{4 \pi} \frac{e^{-j k^{o} r}}{r} \iint_{S} \overline{\overline{\mathbf{G}}}^{\mathbf{A J}}\left(\theta, \phi ; \mathbf{z}^{\prime}\right) \cdot \boldsymbol{J}_{\mathbf{t}}\left(\boldsymbol{r}^{\prime}\right) \mathbf{e}^{\mathbf{j} \mathbf{k}^{\circ} \hat{\boldsymbol{r}} \cdot \overline{\boldsymbol{\rho}}^{\prime}} \mathbf{d} \mathbf{S}^{\prime} \\
& \boldsymbol{F}=\frac{\epsilon_{r}^{o} \epsilon_{0}}{4 \pi} \frac{e^{-j k^{o} r}}{r} \iint_{S} \overline{\overline{\mathbf{G}}} \\
&
\end{aligned}
$$

in which primed coordinates denote the positions on the surface $S, \hat{\boldsymbol{r}}$ is the unit vector in the direction of observation, $k^{\circ}=$ $\omega\left(\epsilon_{r}^{o} \mu_{r}^{o} \epsilon_{0} \mu_{0}\right)^{1 / 2}$ is the wavenumber in the same direction, $\overline{\boldsymbol{\rho}}^{\prime}$ is the lateral position vector $x^{\prime} \hat{\boldsymbol{x}}+y^{\prime} \hat{\boldsymbol{y}}$, and $d S^{\prime}$ is the differential area element. The functions $\overline{\overline{\mathbf{G}}}^{\mathbf{A}}$ and $\overline{\overline{\mathbf{G}}}^{\mathbf{F M}}$ are the asymptotic $(r \rightarrow \infty)$ forms of the dyadic Green's functions pertaining to the specific layered geometry. The $(\cdot)$ operator denotes the dyadic-vector dot product, which produces a vector from another vector. The axial coordinate $z^{\prime}$ does not appear in the phase term in (5)-(6), since the effect of $z^{\prime}$ on the far field is entirely absorbed in the dyadic Green's functions $\overline{\overline{\mathbf{G}}}{ }^{\mathbf{A J}}$ and $\overline{\overline{\mathbf{G}}}{ }^{\mathbf{F M}}$. For free space, a quick comparison with well-known expressions reveals that $\overline{\overline{\mathbf{G}}}^{\mathbf{A J}}$ and $\overline{\overline{\mathbf{G}}}{ }^{\mathbf{F M}}$ are proportional to the identity dyadic $\overline{\bar{I}}$ :

$$
\overline{\overline{\mathbf{G}}}^{\mathbf{A J}}=\overline{\overline{\mathbf{G}}}^{\mathbf{F M}}=\overline{\overline{\mathbf{I}}} \exp \left(\mathbf{j} \mathbf{k}^{\mathbf{o}} \mathbf{z}^{\prime} \cos \theta\right) .
$$

The integrals (5)-(6) with (7) form the basis of the well-known free-space NFFFT [3]. Solving for the vector potentials in general layered media requires much more effort. Our approach is 
based on expressing the radiated field in an entirely different form and comparing the results with (3)-(4). In Appendix A, the radiated field in the upper and lower half spaces is expressed in terms of one-dimensional scalar transmission-line Green's functions [10], [11] by constructing an analogy between Maxwell's equations simplified by planar invariance and transmission-line voltage/current equations. The interested reader is referred to [7] for a more detailed discussion. The necessary information for expressing the vector potentials $\boldsymbol{A}, \boldsymbol{F}$ in terms of the currents $\boldsymbol{J}_{t}, \boldsymbol{M}_{t}$ is contained in Appendix A, (47), (49), (61), and (62). By comparison of these equations with (3)-(6), the dyadic Green's functions $\overline{\overline{\mathbf{G}}}^{\mathrm{AJ}}$ and $\overline{\overline{\mathbf{G}}}^{\mathrm{FM}}$ are found to be

$$
\begin{aligned}
\overline{\overline{\mathbf{G}}}^{\mathbf{A J}}= & \pm\left\{\hat{\boldsymbol{\theta}} \hat{\boldsymbol{x}} \frac{V_{i}^{e}}{\eta^{e}} \cos \phi+\hat{\boldsymbol{\theta}} \hat{\boldsymbol{y}} \frac{V_{i}^{e}}{\eta^{e}} \sin \phi-\hat{\boldsymbol{\theta}} \hat{z} V_{v}^{e} \frac{\epsilon_{r}^{o}}{\epsilon_{r}^{\prime}} \sin \theta\right. \\
& \left.+\cos \theta\left[-\hat{\boldsymbol{\phi}} \hat{\boldsymbol{x}} \frac{V_{i}^{h}}{\eta^{h}} \sin \phi+\hat{\boldsymbol{\phi}} \hat{\boldsymbol{y}} \frac{V_{i}^{h}}{\eta^{h}} \cos \phi\right]\right\} \\
\overline{\overline{\mathbf{G}}}^{\mathbf{F M}}= & \pm\left\{\cos \theta\left[\hat{\boldsymbol{\theta}} \hat{\boldsymbol{x}} V_{v}^{h} \cos \phi+\hat{\boldsymbol{\theta}} \hat{\boldsymbol{y}} V_{v}^{h} \sin \phi-\hat{\boldsymbol{\theta}} \hat{\boldsymbol{z}} \frac{V_{i}^{h}}{\eta^{h}} \frac{\mu_{r}^{o}}{\mu_{r}^{\prime}} \sin \theta\right]\right. \\
& \left.-\hat{\boldsymbol{\phi}} \hat{\boldsymbol{x}} V_{v}^{e} \sin \phi+\hat{\boldsymbol{\phi}} \hat{\boldsymbol{y}} V_{v}^{e} \cos \phi\right\} .
\end{aligned}
$$

Here, $\epsilon_{r}^{\prime}=\epsilon_{r_{n}}-j \sigma_{n} /\left(\omega \epsilon_{0}\right)$ and $\mu_{r}^{\prime}=\mu_{r_{n}}$ denote the complex relative permittivity and the relative permeability at the source point in the $n^{\text {th }}$ layer. The positive and negative signs in front of the curly braces correspond to observation directions in the upper and lower half spaces, respectively. The scalar functions $V_{v}^{p}\left(z^{\prime}\right), V_{i}^{p}\left(z^{\prime}\right)$ are related in a trivial manner to the transmission-line voltage/voltage and voltage/current Green's functions $y_{v}^{p}\left(z \mid z^{\prime}\right), \Upsilon_{i}^{p}\left(z \mid z^{\prime}\right)$ (see Appendix A):

$$
V_{v, i}^{p}\left(z^{\prime}\right)=2 y_{v, i}^{p}\left(\xi \mid z^{\prime}\right) e^{j k^{\circ} \xi \cos \theta} .
$$

As explained in Appendix A, $y_{v}^{p}\left(\xi \mid z^{\prime}\right)$ and $y_{i}^{p}\left(\xi \mid z^{\prime}\right)$ are the voltage responses at $z=\xi$ to an excitation at $z=z^{\prime}$ in the form of an impulsive voltage and current, respectively. Here, $\xi$ is an arbitrary $z$ position that is above (or below, for an observation direction in the lower half space) all the radiators, scatterers, sources, and layer interfaces. It is a temporary value that will be seen to eventually drop out of the equations. The $\theta$ dependence of $V_{v}^{p}\left(z^{\prime}\right), V_{i}^{p}\left(z^{\prime}\right)$ is omitted from the notation for brevity. We will refer to $V_{v}^{p}\left(z^{\prime}\right)$ and $V_{i}^{p}\left(z^{\prime}\right)$ as the transmission-line voltage and current responses, respectively. The parameters $V_{v}^{p}\left(z^{\prime}\right), V_{i}^{p}\left(z^{\prime}\right)$ are the only part of the formulation that depends on the specific layering. In the following, we describe a straightforward method for computing these functions for an arbitrary stratification.

\section{B. Calculation of the Voltage and Current Responses}

From a theoretical standpoint, it turns out to be more convenient to calculate the response inside the layers of a transmission line to an excitation away from all the layer interfaces, rather than the converse implied in (10). The former is strictly analogous to plane-wave incidence on a planar layered medium; a subject that has been documented thoroughly in the literature
[12]. Using the reciprocity properties of the transmission-line equations [10], the observation and excitation coordinates can be exchanged as follows:

$$
\begin{aligned}
& \mathscr{Y}_{i}^{p}\left(\xi \mid z^{\prime}\right)=\mathscr{Y}_{i}^{p}\left(z^{\prime} \mid \xi\right) \\
& \mathscr{y}_{v}^{p}\left(\xi \mid z^{\prime}\right)=-\mathscr{Y}_{i}^{p}\left(z^{\prime} \mid \xi\right) .
\end{aligned}
$$

The Green's functions on the right side now represent the responses at the source point $z^{\prime}$ to a impulsive voltage or current at the auxiliary position $z=\xi$ away from all the sources and layer interfaces. The voltage functions in (10) can therefore be written as

$$
\begin{aligned}
& V_{i}^{p}\left(z^{\prime}\right)=2{ }_{i}^{p}\left(z^{\prime} \mid \xi\right) e^{j k^{\circ} \xi \cos \theta} \\
& V_{v}^{p}\left(z^{\prime}\right)=-2 \mathscr{\nearrow}_{i}^{p}\left(z^{\prime} \mid \xi\right) e^{j k^{o} \xi \cos \theta} .
\end{aligned}
$$

From here on, we will omit the polarization superscript $p$, because the formulas are exactly the same for the two polarizations $e$ and $h$ except the definitions of $\eta^{e}$ and $\eta^{h}$ [see (59)]. The common equations for both polarizations will be presented, with the implicit assumption that the calculation should be done for the TM $(e)$ and TE $(h)$ polarizations separately.

Since (13)-(14) are in the form of voltage and current waves traveling on a transmission line, we can assume that in the $n^{\text {th }}$ layer (see Fig. 1), they are a sum of upward and downward propagating waves:

$$
\begin{aligned}
V_{i_{n}}\left(z^{\prime}\right)= & V_{i_{n}}^{+} e^{-j \beta_{n}\left(z^{\prime}-z_{n}\right)}+V_{i_{n}}^{-} e^{j \beta_{n}\left(z^{\prime}-z_{n-1}\right)} \\
V_{v_{n}}\left(z^{\prime}\right)= & -\frac{V_{i_{n}}^{+}}{\eta_{n}} e^{-j \beta_{n}\left(z^{\prime}-z_{n}\right)}+\frac{V_{i_{n}}^{-}}{\eta_{n}} e^{j \beta_{n}\left(z^{\prime}-z_{n-1}\right)} \\
& (n=0 \ldots N-1),
\end{aligned}
$$

where the upward and downward propagating waves are marked by the superscripts $(+)$ and $(-)$, respectively. Although the interface coordinates $z_{-1}$ and $z_{N-1}$ do not exist, they cancel out in the final equations, so their values can be chosen arbitrarily (for example, $z_{-1}=z_{0}$ and $z_{N-1}=z_{N-2}$ ). They are included in the formulation to preserve uniformity in the notation. The propagation constant $\beta_{n}$ and the intrinsic impedance $\eta_{n}$ in the $n^{\text {th }}$ layer are given by (58) and (59) with (53). As argued in the discussion following (59), the correct root for the propagation constants $\beta_{n}$ is the one with a negative imaginary component; for the exponential term would otherwise grow unphysically without bound in a lossy layer $\left(\sigma_{n} \neq 0\right)$ or in the total-internalreflection (TIR) regime $\left(\Re\left\{\epsilon_{r_{n}}^{\prime} \mu_{r_{n}}^{\prime}\right\}<\epsilon_{r}^{o} \mu_{r}^{o} \sin ^{2} \theta\right)$. With this choice for $\beta_{n}$, the amplitudes of the exponentials in (15)-(16) always remain smaller than unity, since $z_{n}<z^{\prime}<z_{n-1}$ in the $n^{\text {th }}$ layer. This will lead to a numerically stable algorithm for $V_{\{i, v\}_{n}}^{+}, V_{\{i, v\}_{n}}^{-}$that avoids floating-point overflows even for the lossiest layers.

As a first step toward a recursive relation for $V_{i_{n}}^{+}, V_{i_{n}}^{-}$, we introduce the total-wave impedance $Z_{n}$ at the interfaces of the transmission line, defined as the ratio of the total voltage and the total current at $z=z_{n}$ :

$$
Z_{n}=\frac{\mathscr{Y}_{i}\left(z_{n} \mid \xi\right)}{\mathscr{S}_{i}\left(z_{n} \mid \xi\right)}, \quad n=0 \ldots(N-2) .
$$


Eavluating the above expression in both sides of the interface and using the continuity of the voltage and the current, the following recursive relations are obtained for $Z_{n}$ [12], [13]:

$$
\begin{aligned}
Z_{n-1} & =\eta_{n} \frac{Z_{n}-j \eta_{n} \tan \left(\beta_{n} d_{n}\right)}{\eta_{n}-j Z_{n} \tan \left(\beta_{n} d_{n}\right)}, n=N-3 \ldots 0 \\
Z_{n} & =\eta_{n} \frac{Z_{n-1}+j \eta_{n} \tan \left(\beta_{n} d_{n}\right)}{\eta_{n}+j Z_{n-1} \tan \left(\beta_{n} d_{n}\right)}, n=1 \ldots N-2,
\end{aligned}
$$

where $d_{n}=z_{n-1}-z_{n}$ is the (always positive) thickness of the $n^{\text {th }}$ layer. The upward recursion in (18) is useful when the observation direction is in the upper half space; in which case the recursion is initiated at the lowermost interface $\left(z=z_{N-2}\right)$ with

$$
Z_{N-2}=-\eta_{N-1}
$$

This is because there are only downward-propagating waves in the lowermost layer. If the observation direction is in the lower half space, the downward recursion (19) should be used with the following initial value at the uppermost interface $\left(z=z_{0}\right)$ :

$$
Z_{0}=\eta_{0}
$$

As the second step, we derive a relationship between the coefficients $V_{i_{n}}^{+}, V_{i_{n}}^{-}$of the upward and downward propagating waves in terms of the total-wave impedance. Substituting (13)-(16) into (17) and evaluating at $z_{n}$ and $z_{n-1}$, we obtain, respectively,

$$
\begin{aligned}
V_{i_{n}}^{+} & =V_{i_{n}}^{-} \frac{Z_{n}+\eta_{n}}{Z_{n}-\eta_{n}} e^{-j \beta_{n} d_{n}} \\
V_{i_{n}}^{-} & =V_{i_{n}}^{+} \frac{Z_{n-1}-\eta_{n}}{Z_{n-1}+\eta_{n}} e^{-j \beta_{n} d_{n}} .
\end{aligned}
$$

Finally, the continuity of the voltage wave (15) at the interface $z=z_{n-1}$ dictates that

$$
V_{i_{n-1}}^{+}+V_{i_{n-1}}^{-} e^{-j \beta_{n-1} d_{n-1}}=V_{i_{n}}^{+} e^{-j \beta_{n} d_{n}}+V_{i_{n}}^{-} .
$$

A downward recursion for $V_{i_{n}}^{-}$is obtained (after considerable algebra) by substituting (22) into (24), and expressing $Z_{n}$ in terms of $Z_{n-1}$ using (19):

$$
V_{i_{n}}^{-}=V_{i_{n-1}}^{-} \frac{Z_{n-1}-\eta_{n}}{Z_{n-1}-\eta_{n-1}} e^{-j \beta_{n-1} d_{n-1}} .
$$

Similarly, an upward recursion is obtained for $V_{i_{n}}^{+}$by substituting (23) into (24), and expressing $Z_{n-2}$ in terms of $Z_{n-1}$ using (18):

$$
V_{i_{n-1}}^{+}=V_{i_{n}}^{+} \frac{Z_{n-1}+\eta_{n-1}}{Z_{n-1}+\eta_{n}} e^{-j \beta_{n} d_{n}} .
$$

The downward recursion (25) is useful if the observation direction is in the upper half space; in which case, $V_{i_{0}}^{-}$is fixed by the transmission-line excitation at $z=\xi$. It follows from the transmission-line (54)-(55) and symmetry considerations that an impulsive current at $z=\xi$ creates two voltage waves of amplitude $\eta^{p} / 2$ traveling in opposite directions. Therefore, from (15) we can write the following:

$$
V_{i_{0}}^{-} e^{j \beta_{0}\left(z^{\prime}-z_{-1}\right)}=2\left(\frac{\eta_{0}}{2} e^{-j \beta_{0}\left(\xi-z^{\prime}\right)}\right) e^{j k^{\circ} \xi \cos \theta}
$$

which, since $\beta_{0}=k^{o} \cos \theta$ in the upper half space, simplifies to

$$
V_{i_{0}}^{-}=\eta_{0} e^{j \beta_{0} z_{-1}}
$$

Using this as the starting point, $V_{i_{n}}^{-}$in each layer can be calculated recursively using (25). If the observation direction is in the lower half space, the upward recursion (26) for $V_{i_{n}}^{+}$should be used. It is initialized by $V_{i_{N-1}}^{+}$, which satisfies

$V_{i_{N-1}}^{+} e^{-j \beta_{N-1}\left(z^{\prime}-z_{N-1}\right)}=2\left(\frac{\eta_{N-1}}{2} e^{-j \beta_{N-1}\left(z^{\prime}-\xi\right)}\right) e^{j k^{o} \xi \cos \theta}$.

Since $\beta_{N-1}=-k^{o} \cos \theta$ in the lower half space, this amounts to

$$
V_{i_{N-1}}^{+}=\eta_{N-1} e^{-j \beta_{N-1} z_{N-1}} .
$$

The fictitious layer interface positions $z_{-1}$ and $z_{N-1}$ in (28) and (30) are canceled out by the fictitious thicknesses $d_{0}$ and $d_{N-1}$ in the recursions (25)-(26). Their exact values are, therefore, completely arbitrary.

Some of the above formulation bears resemblance to previous results in the literature [4], [13]; although our presentation is more concise (for example, compare (25) with its counterpart $[4,(18 a)])$, numerically more stable, and applicable to any observation angle. The reason behind the numerical stability of our method is that the exponential terms are always bounded in all of the recursive relations, regardless of the conductivities in the layers. In all the recursive relations, the exponentials are in the form $\exp \left(-j \beta_{n} d_{n}\right)$ where $d_{n}$ is a positive real number and the imaginary part of $\beta_{n}$ is zero or negative.

If the far field with respect to a given origin is known, the far field with respect to another origin follows trivially. If the new and old origins are at $\left(x_{1}, y_{1}, z_{1}\right)$ and $\left(x_{0}, y_{0}, z_{0}\right)$, respectively, the far field amplitude $E^{\prime}$ with respect to the new origin is simply a phase-shifted version of the far field amplitude $E$ with respect to the old origin;

$$
\begin{aligned}
E^{\prime}=E \exp [ & -j k^{o}\left(\left(x_{1}-x_{0}\right) \cos \phi \sin \theta\right. \\
& \left.\left.+\left(y_{1}-y_{0}\right) \sin \phi \sin \theta+\left(z_{1}-z_{0}\right) \cos \theta\right)\right] .
\end{aligned}
$$

\section{Explicit Formulas for Three Layers}

For up to three layers, compact closed-form expressions for $V_{v}\left(z^{\prime}\right)$ and $V_{i}\left(z^{\prime}\right)$ can be found by explicitly carrying out the recursions in the previous subsection. If the observation direction is in the upper half space $\left(\epsilon_{r}^{o}=\epsilon_{r_{0}}\right)$,

$$
V_{v_{0}}\left(z^{\prime}\right)=e^{j \beta_{0} z^{\prime}}-\Gamma_{01} e^{-j \beta_{0} z^{\prime}}-\frac{\Upsilon_{01} \Gamma_{12} \Upsilon_{10} e^{-j\left(\beta_{0} z^{\prime}+2 \beta_{1} d_{1}\right)}}{1-\Gamma_{10} \Gamma_{12} e^{-j 2 \beta_{1} d_{1}}}
$$


TABLE I

$(x, y, z)$ Positions of the Hertzian Dipoles Used as Excitation IN THE EXAMPLE PROBLEM (UNITS IN MM).

\begin{tabular}{|l|l|l|}
\hline$x$-directed & $y$-directed & $z$-directed \\
\hline$(19.95,19,-47.5)$ & $(0,0.95,-47.5)$ & $(-19,-19,-46.55)$ \\
\hline$(19.95,19,0)$ & $(0,0.95,0)$ & $(-19,-19,0.95)$ \\
\hline$(19.95,19,47.5)$ & $(0,0.95,47.5)$ & $(-19,-19,48.45)$ \\
\hline
\end{tabular}

$$
\begin{aligned}
& V_{v_{1}}\left(z^{\prime}\right)=\Upsilon_{10} \frac{e^{j \beta_{1} z^{\prime}}-\Gamma_{12} e^{-j \beta_{1}\left(2 d_{1}+z^{\prime}\right)}}{1-\Gamma_{10} \Gamma_{12} e^{-j 2 \beta_{1} d_{1}}} \\
& V_{v_{2}}\left(z^{\prime}\right)=\frac{\Upsilon_{21} \Upsilon_{10} e^{j\left(\beta_{2}\left(z^{\prime}+d_{1}\right)-\beta_{1} d_{1}\right)}}{1-\Gamma_{10} \Gamma_{12} e^{-j 2 \beta_{1} d_{1}}} .
\end{aligned}
$$

If the observation direction is in the lower half space $\left(\epsilon_{r}^{o}=\epsilon_{r_{2}}\right)$,

$$
\begin{aligned}
V_{v_{0}}\left(z^{\prime}\right)= & -\frac{\Upsilon_{01} \Upsilon_{12} e^{-j\left(\beta_{0} z^{\prime}+\beta_{1} d_{1}-\beta_{2} d_{1}\right)}}{1-\Gamma_{10} \Gamma_{12} e^{-j 2 \beta_{1} d_{1}}} \\
V_{v_{1}}\left(z^{\prime}\right)= & -\frac{\Upsilon_{12} e^{-j\left(\beta_{1} d_{1}-\beta_{2} d_{1}\right)}}{1-\Gamma_{10} \Gamma_{12} e^{-j 2 \beta_{1} d_{1}}}\left[e^{-j \beta_{1} z^{\prime}}-\Gamma_{10} e^{j \beta_{1} z}\right] \\
V_{v_{2}}\left(z^{\prime}\right)= & -e^{-j \beta_{2} z^{\prime}}+\Gamma_{21} e^{j \beta_{2}\left(z^{\prime}+2 d_{1}\right)} \\
& +\frac{\Upsilon_{21} \Gamma_{10} \Upsilon_{12} e^{j\left(\beta_{2}\left(z^{\prime}+2 d_{1}\right)-\beta_{1} 2 d_{1}\right)}}{1-\Gamma_{10} \Gamma_{12} e^{-j 2 \beta_{1} d_{1}}} .
\end{aligned}
$$

In the above, the reflection and transmission coefficients $\Gamma_{m n}$, $\Upsilon_{m n}$ are defined as follows:

$$
\Gamma_{m n}=\Upsilon_{m n}-1=\frac{\eta_{m}-\eta_{n}}{\eta_{m}+\eta_{n}}, \quad n=0,1,2
$$

The current responses $V_{i_{n}}\left(z^{\prime}\right)$ can be obtained trivially from $V_{v_{n}}\left(z^{\prime}\right)$ using the relations (15)-(16). The downward-propagating parts of $V_{v_{n}}\left(z^{\prime}\right)$ with $\exp (j \beta z)$ dependence are multiplied by $\eta_{n}$, whereas the upward-propagating parts with $\exp (-j \beta z)$ dependence are multiplied by $-\eta_{n}$.

For free space $\left(\epsilon_{r_{0}}=\epsilon_{r_{1}}=\epsilon_{r_{2}}, \mu_{r_{0}}=\mu_{r_{1}}=\mu_{r_{2}}\right)$, $V_{v}^{e}\left(z^{\prime}\right)$ and $V_{v}^{h}\left(z^{\prime}\right)$ reduce to $\exp \left(j k^{o} z^{\prime} \cos \theta\right), V_{i}^{e}\left(z^{\prime}\right)$ reduces to $\eta^{e} \exp \left(j k^{o} z^{\prime} \cos \theta\right) \cos \theta$, and $V_{i}^{h}\left(z^{\prime}\right)$ reduces to $\eta^{h} \exp \left(j k^{\circ} z^{\prime} \cos \theta\right) / \cos \theta$. Substituting these into (8)-(9), it is easily found that these dyadics both reduce to (7), leading to the well-known free-space formulation [3].

\section{NUMERICAL RESULTS}

In this section, we will demonstrate the accuracy of the NFFFT algorithm in the previous section by comparing the numerical results obtained using a finite-difference time-domain (FDTD) implementation with theoretical values. An 8-layered space is considered for our example, with layer thicknesses $d_{1}=d_{2}=$ $\ldots=d_{6}=1.9 \mathrm{~cm}$. The relative permittivities, relative permeabilities and the electrical conductivities are varied linearly from $\epsilon_{r_{0}}=1.3 \rightarrow \epsilon_{r_{7}}=1.5, \mu_{r_{0}}=1.1 \rightarrow \mu_{r_{7}}=1.3$, and $\sigma_{1}=0.1 \mathrm{~S} / \mathrm{m} \rightarrow \sigma_{r_{6}}=0.35 \mathrm{~S} / \mathrm{m}$. The upper and lower half spaces are assumed lossless: $\sigma_{0}=\sigma_{r_{7}}=0$. The origin is placed at the uppermost surface of the layering structure. A total of 9 infinitesimal Hertzian dipoles are placed in the FDTD grid at the positions with respect to the origin indicated in Table I. The
Hertzian dipoles are modeled in the FDTD method by a single current element on the edge of a grid cell. Each dipole represents a current distribution of the form

$$
\boldsymbol{J}(\boldsymbol{r})=\hat{\boldsymbol{a}} p(t) \delta\left(x-x_{0}\right) \delta\left(y-y_{0}\right) \delta\left(z-z_{0}\right)
$$

where $\hat{\boldsymbol{a}}$ is either $\hat{\boldsymbol{x}}, \hat{\boldsymbol{y}}$, or $\hat{\boldsymbol{z}}, \delta(\cdot)$ is the Dirac delta function, and the dipole moment $p(t)$ is a sine-modulated Gaussian waveform:

$$
p(t)=\sin \left(2 \pi f_{0} t\right) e^{-t^{2} / 2 \tau^{2}}
$$

with $f_{0}=6 \mathrm{GHz}$ and $\tau=0.242 \mathrm{~ns}$. The power in the corresponding temporal spectrum $(\omega>0)$

$$
\tilde{p}(\omega)=-j \sqrt{\frac{\pi}{2}} \tau e^{-\left(\omega-2 \pi f_{0}\right)^{2} \tau^{2} / 2}
$$

falls to $-40 \mathrm{~dB}$ of its maximum at $4 \mathrm{GHz}$ and $8 \mathrm{GHz}$. The theoretical far field created by the dipoles can be found using the formulation in the previous section. For example, the radiated electric field of a time-harmonic $z$-directed Hertzian dipole of current moment $\tilde{p}(\omega)$ placed at $\left(0,0, z_{0}\right)$ is, from (3), (5) and (8),

$$
E_{\theta}=j \omega \frac{\mu_{r}^{o} \mu_{0}}{4 \pi} \frac{e^{-j k r}}{r} \tilde{p}(\omega) V_{v_{0}}^{e}\left(z_{0}\right) \frac{\epsilon_{r}^{o}}{\epsilon_{r_{0}}} \sin \theta
$$

in which $\epsilon_{r}^{o}$ is the relative permittivity of the observation half space and $V_{v_{n}}^{e}\left(z^{\prime}\right)$ is given by (16). The parameters of the FDTD simulation are the following: grid dimensions $15.2 \mathrm{~cm} \times 15.2 \mathrm{~cm} \times 15.2$ $\mathrm{cm}$, grid spacing $\Delta x=\Delta y=\Delta z=\Delta=1.9 \mathrm{~mm}$, time step $\Delta t=(0.98 / \sqrt{3}) \Delta / c$. The computational grid is truncated by 10-cell thick convolution perfectly matched layer (CPML) [14]. The constitutive parameters $\epsilon$ and $\mu$ are interpolated at the planar interfaces to preserve second-order accuracy [15]. The scattered field is collected on a rectangular NFFFT surface 3 cells away from the PML, and transformed to the far field using the NFFFT described in Section II. For better accuracy, two NFFFT surfaces are used for $\boldsymbol{J}_{t}$ and $\boldsymbol{M}_{t}$ [16], and the wavenumber $k^{o}$ is dispersion-corrected using the exact dispersion relation in the observation half space [3]. All far field amplitudes are normalized by the factor

$$
E_{0}(\omega)=j \omega \frac{\mu_{0}}{4 \pi} \frac{e^{-j k r}}{r}\left[-j \sqrt{\frac{\pi}{2}} \tau e^{-\left(\omega-2 \pi f_{0}\right)^{2} \tau^{2} / 2}\right]
$$

which is the broadside amplitude of the far field created by a dipole with time-harmonic current moment (41) in free space. In Fig. 2(a), the real and imaginary parts of both the $\theta$ and $\phi$ components of the electric field at $\theta=\phi=45^{\circ}$ are compared with theoretical results for 100 equally-spaced frequencies between 4 $\mathrm{GHz}$ and $8 \mathrm{GHz}$. The solid and dashed lines represent the FDTD results, while the dot and cross marks denote theoretical values. In Fig. 2(b), the $\theta$ and $\phi$ components of the phasor electric field at $f=6 \mathrm{GHz}$ on the $\phi=45^{\circ}$ plane is shown on a Cartesian plot at 240 equally-spaced $\theta$ values between 0 and $2 \pi$ (end points included). The angles $\theta=\pi / 2$ and $\theta=3 \pi / 2$ are deliberately not included in this range, since there are singularities in the formulas at these angles. However, the formulas remain stable for angles arbitrarily close to $\theta=\pi / 2$ and $\theta=3 \pi / 2$. In Fig. 2(c), the $\theta$ and $\phi$ components of the phasor electric field at $f=6 \mathrm{GHz}$ on the cone $\theta=135^{\circ}$ is shown in a Cartesian plot at 120 equally-spaced 

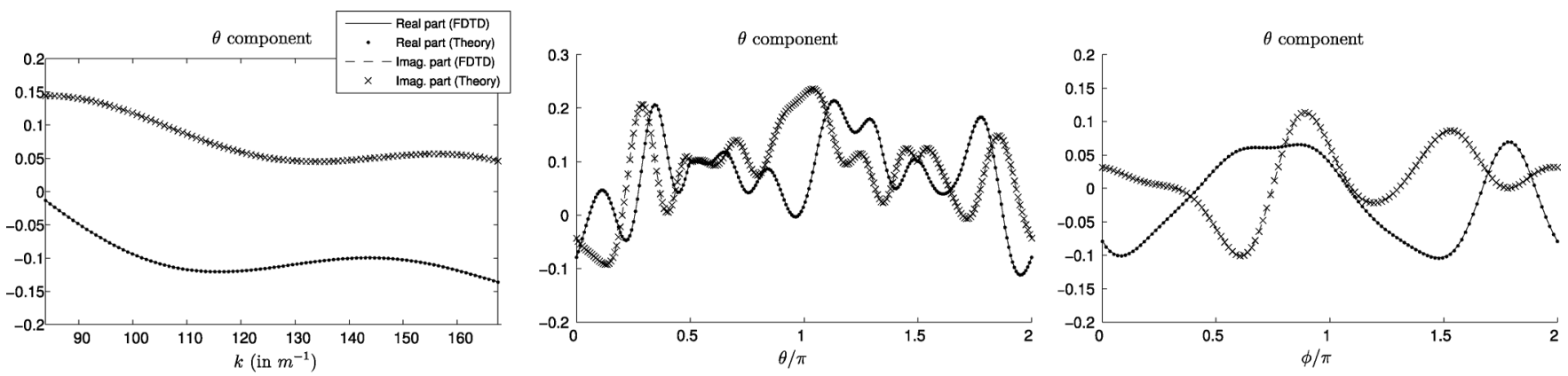

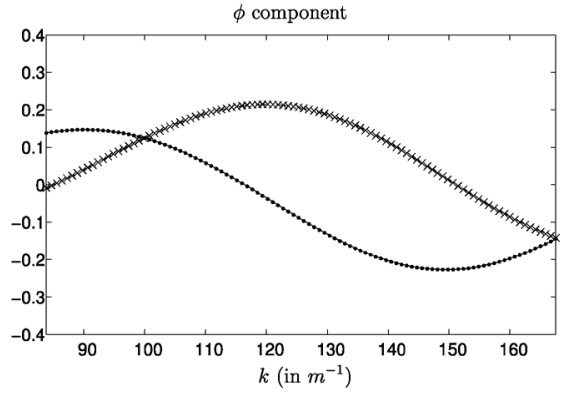

(a)

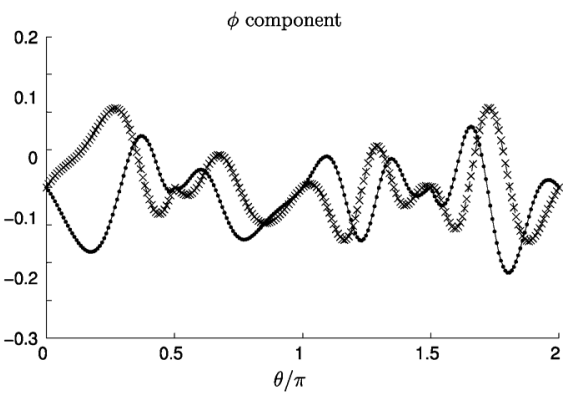

(b)

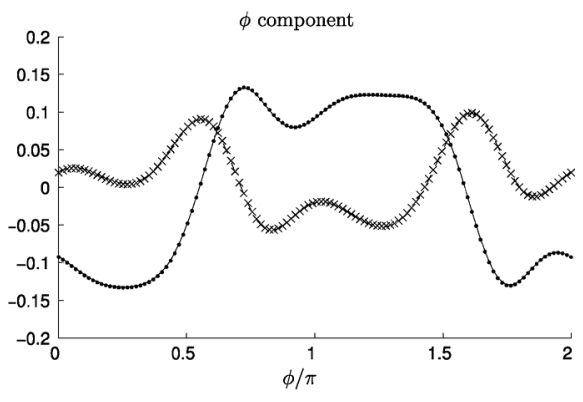

(c)

Fig. 2. Comparison of phasor far-field amplitudes obtained using FDTD with theoretical values for a total of 9 Hertzian dipoles radiating in an 8-layered medium. The positions of the dipoles are given in Table I. (a) Frequency spectra of $E_{\theta}$ (top) and $E_{\phi}$ (bottom) at $\theta=\phi=45^{\circ}$. (b) Cartesian plot of $E_{\theta}$ (top) and $E_{\phi}$ (bottom) with respect to $\theta / \pi$ on the $\phi=45^{\circ}$ plane at $f=6 \mathrm{GHz}$. (c) Cartesian plot of $E_{\theta}$ (top) and $E_{\phi}$ (bottom) with respect to $\phi$ at $\theta=135^{\circ}$ and $f=6 \mathrm{GHz}$. The legend in (a) applies to all subfigures.

$\phi$ values between 0 and $2 \pi$ (end points included). The maximum root-mean-square error in all the plots in Fig. 2 is $1 \%$.

\section{SOFTWARE IMPLEMENTATION}

A general-purpose finite-difference time-domain (FDTD) software package is being developed by the Biophotonics Group in the Biomedical Engineering Department of Northwestern University. The software is developed as part of National Institutes of Health (NIH) grants R01EB003682 and R01CA128641, and will be made freely available to the public in open source. It features a full implementation of the NFFFT algorithm described in this paper, as well as other auxiliary methods pertaining to layered media that are not currently available in commercial FDTD packages. Interested readers can contact capoglu@ieee.org to be notified when the software becomes available for download.

\section{SUMMARY}

In this paper, a concise and numerically stable frequency-domain near-field-to-far-field transform (NFFFT) is described for general planar layered media. The results are applicable to layers with arbitrary frequency-dispersion properties, although only electrical conductivity is considered here. Unlike previous studies, the formulation also covers observation angles in the lower half space. The results are especially relevant to differential-equation-based finite methods such as the finite-difference time-domain (FDTD) method and the finite-element method (FEM). Although the core derivation is based on a transmission-line analogy, the final formulas are presented in vector-potential form; which helps the transition from free-space implementations. Numerical FDTD results were given for an 8-layered medium and shown to agree well with theory.

\section{APPENDIX \\ RAdiATED EleCtric Field IN TERMS OF THE TRANSMISSION-LINE GREEN'S FUNCTIONS}

If the electric-field distribution on an infinite planar surface is known and the region above the planar surface is homogeneous, then the electric field above the planar surface is also known [17]. The same principle applies to the electric field in the lower half space, if it is known on a plane situated below all the sources. We will now make use of these facts to derive the radiated electric field in the uppermost and lowermost regions of the multilayered structure shown in Fig. 1. The relative permittivity and permeability in the observation region will be denoted by $\epsilon_{r}^{o}$ and $\mu_{r}^{o}$, respectively. In the notation of Fig. 1(a), these are $\epsilon_{r_{0}}, \mu_{r_{0}}$ if the observation direction is in the upper half space, and $\epsilon_{r_{N-1}}, \mu_{r_{N-1}}$ otherwise.

Let the equivalent electric and magnetic currents $\boldsymbol{J}_{t}\left(\boldsymbol{r}^{\prime}\right)$, $\boldsymbol{M}_{t}\left(\boldsymbol{r}^{\prime}\right)$ radiate in the layered space shown in Fig. 1(b). Let $z=\xi$ be any plane above or below all the layer interfaces and the source currents. The exact value of $\xi$ is immaterial since it will cancel out in the final result; but it is of conceptual importance that it exists. In the subsequent analysis, we will use the plane-wave spectrum of the electric field on this plane to derive the radiated electric field in the upper and lower half spaces. Let the plane-wave spectrum of the 2D electric-field distribution $\boldsymbol{E}(x, y)$ on the planar surface $z=\xi$ be defined by the following Fourier transform operation:

$$
\widetilde{\mathbf{E}}\left(k_{x}, k_{y}, \xi\right)=\iint_{-\infty}^{\infty} \boldsymbol{E}(x, y, \xi) e^{j\left(k_{x} x+k_{y} y\right)} d x d y .
$$

The inverse of this Fourier relation is the plane-wave representation

$$
\boldsymbol{E}(x, y, \xi)=\frac{1}{(2 \pi)^{2}} \iint_{-\infty}^{\infty} \widetilde{\mathbf{E}}\left(k_{x}, k_{y}, \xi\right) e^{-j\left(k_{x} x+k_{y} y\right)} d k_{x} d k_{y}
$$


In the above, the Fourier operation is applied separately to each Cartesian component of the electric-field vector. Extending each plane wave in (45) into the upper or lower half space, the following expression can be written for the electric field anywhere in the region $z>\xi$ or $z<\xi$ :

$$
\begin{aligned}
\boldsymbol{E}(x, y, z)=\frac{1}{(2 \pi)^{2}} \int & \int_{-\infty}^{\infty} \widetilde{\mathbf{E}}\left(k_{x}, k_{y}, \xi\right) \\
& \times e^{-j\left(k_{x} x+k_{y} y \pm k_{z}(z-\xi)\right)} d k_{x} d k_{y},
\end{aligned}
$$

with $k_{z}=\left(\left(k^{o}\right)^{2}-k_{x}^{2}-k_{y}^{2}\right)^{1 / 2}$, and $k^{o}=(\omega / c)\left(\epsilon_{r}^{o} \mu_{r}^{o}\right)^{1 / 2}$ is the wavenumber in the observation half space. The plus and minus signs in front of $k_{z}$ are for the upper and lower half spaces, respectively. This field satisfies Maxwell's equations in the observation region, as well as the boundary conditions at $z=\xi$ specified by (45). It is therefore the unique solution for the electric field in the observation region. The radiated electric field, defined as the asymptotic limit of the integral (46) for $r \rightarrow \infty$, can be obtained using the steepest-descent method [17]:

$$
\boldsymbol{E}_{r}(r, \theta, \phi)=\frac{e^{-j k^{o} r}}{r} \frac{j k^{o}|\cos \theta|}{2 \pi} e^{j k^{\circ} \xi \cos \theta} \widetilde{\mathbf{E}}(\alpha, \beta, \xi)
$$

in which $(r, \theta, \phi)$ are the usual spherical coordinates, and

$$
(\alpha, \beta)=\left(k^{o} \cos \phi \sin \theta, k^{o} \sin \phi \sin \theta\right) .
$$

The far-field expression (47) is valid for the full $\theta$ range from 0 to $\pi$, covering observation angles both in the upper and lower half spaces. The interpretation of (47) is that the far-field at a given observation direction is directly proportional to the vector amplitude of the plane-wave component on the surface $z=$ $\xi$ that is incident in the same direction. For a planar layered medium, the invariance of the geometry perpendicular to the axis of stratification (the $z$ axis) can be exploited to obtain the vector plane-wave amplitude $\widetilde{\mathbf{E}}(\alpha, \beta, \xi)$ in terms of one-dimensional scalar transmission-line Green's functions [10], [11]. As a consequence of the lateral invariance of the geometry, the following spectral superposition integral holds for $\widetilde{\mathbf{E}}(\alpha, \beta, \xi)$ [7]:

$$
\begin{aligned}
& \widetilde{\boldsymbol{E}}(\alpha, \beta, \xi)=\iint_{S} \overline{\overline{\mathbf{G}}}^{\mathbf{E J}}\left(\alpha, \beta, \xi \mid \mathbf{z}^{\prime}\right) \cdot \boldsymbol{J}_{\mathbf{t}}\left(\boldsymbol{r}^{\prime}\right) \mathbf{e}^{\mathrm{j} \boldsymbol{k}_{\boldsymbol{\rho}} \cdot \boldsymbol{\rho}^{\prime}} \mathbf{d} \mathbf{S}^{\prime} \\
&+\iint_{S} \overline{\overline{\mathbf{G}}}^{\mathbf{E M}}\left(\alpha, \beta, \xi \mid \mathbf{z}^{\prime}\right) \cdot \boldsymbol{M}_{\mathbf{t}}\left(\boldsymbol{r}^{\prime}\right) \mathbf{e}^{\mathrm{j} \boldsymbol{k}_{\boldsymbol{\rho}} \cdot \boldsymbol{\rho}^{\prime}} \mathbf{d} \mathbf{S}^{\prime},
\end{aligned}
$$

where $\boldsymbol{k}_{\boldsymbol{\rho}}=\alpha \hat{\boldsymbol{x}}+\beta \hat{\boldsymbol{y}}$ and $\boldsymbol{\rho}^{\prime}=x^{\prime} \hat{\boldsymbol{x}}+y^{\prime} \hat{\boldsymbol{y}}$ is the lateral position vector at the source point. In (49), $\overline{\overline{\mathbf{G}}}^{\mathbf{E J}, \mathbf{E M}}\left(\alpha, \beta, \mathbf{z} \mid \mathbf{z}^{\prime}\right)$ denote the spectral dyadic Green's functions, explicit expressions for which are given in [10, Eqs. (28)-(31)]. These expressions are reproduced here for the sake of completeness:

$$
\begin{aligned}
& \widetilde{\bar{G}}^{E J}\left(\alpha, \beta, \xi \mid z^{\prime}\right)=-\hat{\boldsymbol{u}} \hat{\boldsymbol{u}} \gamma_{i}^{e}-\hat{\boldsymbol{v}} \hat{\boldsymbol{v}} \gamma_{i}^{h} \\
& +\hat{z} \hat{\boldsymbol{u}} \frac{k_{\rho}}{\omega \epsilon_{0} \epsilon_{r}^{o}} \mathscr{Y}_{i}^{e}+\hat{\boldsymbol{u}} \hat{z} \frac{k_{\rho}}{\omega \epsilon_{0} \epsilon_{r}^{\prime}} y_{v}^{e} \\
& +\hat{z} \hat{z} \frac{1}{j \omega \epsilon_{0} \epsilon_{r}^{o}}\left[\frac{k_{\rho}^{2}}{j \omega \epsilon_{0} \epsilon_{r}^{\prime}} \mathscr{J}_{v}^{e}-\delta\left(\xi-z^{\prime}\right)\right]
\end{aligned}
$$

$$
\begin{aligned}
\widetilde{\overline{\boldsymbol{G}}}^{E M}\left(\alpha, \beta, \xi \mid z^{\prime}\right)= & -\hat{\boldsymbol{u}} \hat{\boldsymbol{v}} \int_{v}^{e}+\hat{\boldsymbol{v}} \hat{\boldsymbol{u}} \Upsilon_{v}^{h} \\
& +\hat{z} \hat{\boldsymbol{v}} \frac{k_{\rho}}{\omega \epsilon_{0} \epsilon_{r}^{o}} \mathscr{J}_{v}^{e}-\hat{\boldsymbol{v}} \hat{z} \frac{k_{\rho}}{\omega \mu_{0} \mu_{r}^{\prime}} \mho_{i}^{h} .
\end{aligned}
$$

In these expressions, the unit vector $\hat{\boldsymbol{u}}$ is defined to be parallel to the $x y$-projection of the radial unit vector $\hat{r}$ in the direction of observation, while $\hat{\boldsymbol{v}}$ is the $90^{\circ}$-rotation of $\hat{\boldsymbol{u}}$ in the $x y$ plane:

$$
\hat{\boldsymbol{u}}=\cos \phi \hat{\boldsymbol{x}}+\sin \phi \hat{\boldsymbol{y}}, \quad \hat{\boldsymbol{v}}=-\sin \phi \hat{\boldsymbol{x}}+\cos \phi \hat{\boldsymbol{y}} .
$$

The unit vector $\hat{\boldsymbol{v}}$ is equivalent to the unit vector $\hat{\boldsymbol{\phi}}$ in spherical coordinates. In (50)-(51), $k_{\rho}=\left|\boldsymbol{k}_{\boldsymbol{\rho}}\right|=k^{o} \sin \theta$, and

$$
\epsilon_{r}^{\prime}=\epsilon_{r_{n}}-j \frac{\sigma_{n}}{\omega \epsilon_{0}}, \quad \mu_{r}^{\prime}=\mu_{r_{n}}
$$

are the complex relative permittivity and the relative permeability at the source coordinate $z^{\prime}$, assumed to be in the $n^{\text {th }}$ layer. The scalar functions $\mathcal{Y}_{v}^{p}, y_{i}^{p}, \mathscr{I}_{v}^{p}, \mathscr{Y}_{i}^{p}$ (polarization superscript $p$ being $e$ or $h$ ) are transmission-line Green's functions, representing the voltage (or current) response at $z=\xi$ to an impulsive voltage (or current) excitation at the source coordinate $z=z^{\prime}$. They satisfy the following transmission-line equations:

$$
\begin{aligned}
& \frac{\mathrm{d} \mathscr{Y}_{i}^{p}}{\mathrm{~d} z}=-j \beta \eta^{p} \mathscr{Y}_{i}^{p} \\
& \frac{\mathrm{d} \mathscr{Y}_{i}^{p}}{\mathrm{~d} z}=-j \frac{\beta}{\eta^{p}} \mathscr{Y}_{i}^{p}+\delta\left(z-z^{\prime}\right),
\end{aligned}
$$

and

$$
\begin{aligned}
& \frac{\mathrm{d} \gamma_{v}^{p}}{\mathrm{~d} z}=-j \beta \eta^{p} \cdot{ }_{v}^{p}+\delta\left(z-z^{\prime}\right) \\
& \frac{\mathrm{d} \mathscr{\mathscr { Y }} \underset{v}{p}}{\mathrm{~d} z}=-j \frac{\beta}{\eta^{p}} y_{v}^{p},
\end{aligned}
$$

where the propagation constant $\beta$ and the polarization-dependent intrinsic impedance $\eta^{p}$ of the transmission line are

$$
\begin{aligned}
\beta & =k^{o} \sqrt{\frac{\epsilon_{r}^{\prime} \mu_{r}^{\prime}}{\epsilon_{r}^{o} \mu_{r}^{o}}-\sin ^{2} \theta} \\
\eta^{e} & =\eta^{o} \frac{\beta \epsilon_{r}^{o}}{k^{o} \epsilon_{r}^{\prime}}, \quad \eta^{h}=\eta^{o} \frac{k^{o} \mu_{r}^{\prime}}{\beta \mu_{r}^{o}} .
\end{aligned}
$$

In (59), $\eta^{o}=\sqrt{\left(\mu_{r}^{o} \mu_{0}\right) /\left(\epsilon_{r}^{o} \epsilon_{0}\right)}$ is the wave impedance of the observation half space. In lossy layers $\left(\sigma_{n} \neq 0\right)$ or in the totalinternal-reflection (TIR) regime $\left(\Re\left\{\epsilon_{r}^{\prime} \mu_{r}^{\prime}\right\}<\epsilon_{r}^{o} \mu_{r}^{o} \sin ^{2} \theta\right)$, the sign of the imaginary part of the propagation constant $\beta$ should negative so that all the exponentials decay in the direction of propagation.

Thanks to the placement of $\xi$ above (or below) all the sources, $\xi$ never equals $z^{\prime}$; therefore, the term $\delta\left(\xi-z^{\prime}\right)$ in (50) can be neglected. Because $\xi$ is also above (or below) all layer interfaces, there is only an upward- (or downward-) propagating transmission-line voltage/current wave at $z=\xi$. Consequently, the ratio between the transmission-line voltages $y_{i}^{p}, y_{v}^{p}$ and the transmission-line currents $\mathscr{T}_{i}^{p}, \mathscr{Y}_{v}^{p}$ is simply the intrinsic impedance $\eta^{p}$ :

$$
\begin{gathered}
\mathscr{T}_{i}^{p}= \pm \frac{\mathcal{Y}_{i}^{p}}{\eta^{p}}, \quad \mathscr{T}_{v}^{p}= \pm \frac{\mathscr{Y} p}{\eta_{v}^{p}} \\
\eta^{e}= \pm \eta^{o} \cos \theta, \quad \eta^{h}= \pm \eta^{o} / \cos \theta .
\end{gathered}
$$

The positive and negative signs in the above relations correspond to the upper and lower half spaces, respectively. Note that the intrinsic impedance $\eta^{p}$ is defined to be positive, hence the 
\pm sign in front of the $\cos \theta$ term. The dyadic Green's functions are put into a more convenient form if the first vector component in each of the dyads $\hat{\boldsymbol{u}} \hat{\boldsymbol{u}}, \hat{\boldsymbol{u}} \hat{\boldsymbol{v}}, \hat{\boldsymbol{u}} \hat{\boldsymbol{z}}$, etc. is projected onto the spherical-coordinate unit vectors $(\hat{\boldsymbol{r}}, \hat{\boldsymbol{\theta}}, \hat{\boldsymbol{\phi}})$, whereas the second vector component in each dyad is projected onto the Cartesian unit vectors $(\hat{\boldsymbol{x}}, \hat{\boldsymbol{y}}, \hat{z})$. After substituting (60) and simplifying considerably, the following expressions for the dyadic Green's functions are obtained:

$$
\begin{aligned}
& \widetilde{\overline{\boldsymbol{G}}}^{E J}\left(\alpha, \beta, \xi \mid z^{\prime}\right)=-\hat{\boldsymbol{\theta}} \hat{\boldsymbol{x}} \gamma_{i}^{e} \sec \theta \cos \phi-\hat{\boldsymbol{\theta}} \hat{\boldsymbol{y}} \gamma_{i}^{e} \sec \theta \sin \phi \\
& +\hat{\boldsymbol{\theta}} \hat{z} y_{v}^{e} \eta^{o} \frac{\epsilon_{r}^{o}}{\epsilon_{r}^{\prime}} \tan \theta+\hat{\boldsymbol{\phi}} \hat{\boldsymbol{x}} y_{i}^{h} \sin \phi \\
& -\hat{\boldsymbol{\phi}} \hat{\boldsymbol{y}}{ }_{i}^{h} \cos \phi \\
& \widetilde{\widetilde{\boldsymbol{G}}}^{E M}\left(\alpha, \beta, \xi \mid z^{\prime}\right)=\hat{\boldsymbol{\theta}} \hat{\boldsymbol{x}} y_{v}^{e} \sec \theta \sin \phi-\hat{\boldsymbol{\theta}} \hat{\boldsymbol{y}}{ }_{v}^{e} \sec \theta \cos \phi \\
& +\hat{\boldsymbol{\phi}} \hat{\boldsymbol{x}}{ }_{v}^{h} \cos \phi+\hat{\boldsymbol{\phi}} \hat{\boldsymbol{y}}{ }_{v}^{h} \sin \phi \\
& -\hat{\boldsymbol{\phi}} \hat{z} \gamma_{i}^{h} \frac{1}{\eta^{o}} \frac{\mu_{r}^{o}}{\mu_{r}^{\prime}} \sin \theta \text {. }
\end{aligned}
$$

Substituting (61)-(62) into (49), and (49) into (47), the radiated electric field is expressed in terms of scalar transmission-line Green's functions $y_{v}^{p}, y_{i}^{p}, \mathscr{Y}_{v}^{p}, \mathscr{Y}_{i}^{p}$ (polarization superscript $e$ or $h$ ) that satisfy (54)-(56). The calculation of the radiated electric field is thus reduced to the calculation of $\mathscr{\gamma} \underset{v}{p}, \mathscr{q}_{i}^{p}$, $\mathscr{S}_{v}^{p}, \mathscr{Y}_{i}^{p}$ for a given layering structure in the $z$ direction.

\section{REFERENCES}

[1] K. Umashankar and A. Taflove, "A novel method to analyze electromagnetic scattering of complex objects," IEEE Trans. Electromagn. Compat., vol. EMC-24, no. 4, pp. 397-405, Nov. 1982.

[2] A. Taflove and K. Umashankar, "Radar cross section of general three-dimensional scatterers," IEEE Trans. Electromagn. Compat., vol. EMC-25, no. 4, pp. 433-440, Nov. 1983.

[3] A. Taflove and S. C. Hagness, Computational Electrodynamics: The Finite-Difference Time-Domain Method,3rd ed. Boston, MA: Artech House, 2005.

[4] K. Demarest, Z. Huang, and R. Plumb, "An FDTD near-to-far-zone transformation for scatterers buried in stratified grounds," IEEE Trans. Antennas Propag., vol. 44, no. 8, pp. 1150-7, Aug. 1996.

[5] I. R. Capoglu and G. S. Smith, "A direct time-domain FDTD near-field-to-far-field transform in the presence of an infinite grounded dielectric slab," IEEE Trans. Antennas Propag., vol. 54, no. 12, pp. 3805-14, Dec. 2006.

[6] I. R. Capoglu and G. S. Smith, "Application of new tools for the FDTD analysis of UWB antennas on dielectric substrates," Microw. Opt. Technol. Lett., vol. 50, no. 5, pp. 1447-1451, May 2008.

[7] I. R. Capoglu, "Techniques for handling multilayered media in the FDTD method," Ph.D. dissertation, Georgia Inst. Technol., Atlanta, GA, 2007.

[8] C. M. Furse and O. P. Gandhi, "Why the DFT is faster than the FFT for FDTD time-to-frequency domain conversions," IEEE Microw. Guided Wave Lett., vol. 5, no. 10, pp. 326-328, Oct. 1995.

[9] C. A. Balanis, Advanced Engineering Electromagnetics. New York: Wiley, 1989

[10] K. A. Michalski and J. R. Mosig, "Multilayered media Green's functions in integral equation formulations," IEEE Trans. Antennas Propag., vol. 45, no. 3, pp. 508-19, Mar. 1997.
[11] L. B. Felsen and N. Marcuvitz, Radiation and Scattering of Waves. Piscataway, NJ: IEEE Press, 1994.

[12] S. J. Orfanidis, MATLAB Toolbox. Electromagnetic Waves and Antennas - Web Resource. 2007 [Online]. Available: http://www.ece.rutgers.edu/ orfanidi/ewa/

[13] K. Demarest, R. Plumb, and Z. Huang, "FDTD modeling of scatterers in stratified media," IEEE Trans. Antennas Propag., vol. 43, no. 10, pp. 1164-1168, Oct. 1995.

[14] J. A. Roden and S. D. Gedney, "Convolution PML (CPML): An efficient FDTD implementation of the CFD-PML for arbitrary media," Microw. Opt. Technol. Lett., vol. 27, no. 5, pp. 334-9, Dec. 2000.

[15] K.-P. Hwang and A. C. Cangellaris, "Effective permittivities for second-order accurate FDTD equations at dielectric interfaces," IEEE Microw. Wireless Compon. Lett., vol. 11, no. 4, pp. 158-60, Apr. 2001.

[16] T. Martin, "An improved near- to far-zone transformation for the finitedifference time-domain method," IEEE Trans. Antennas Propag., vol. 46, no. 9, pp. 1263-1271, Sep. 1998.

[17] G. S. Smith, An Introduction to Classical Electromagnetic Radiation. New York: Cambridge Univ. Press, 1997.

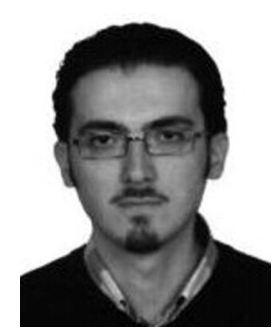

İlker R. Çapoğlu (S'03-M'07) received the Ph.D. degree in electrical and computer engineering from the Georgia Institute of Technology, Atlanta, in 2007.

He has since been employed as a postdoctoral fellow in the Biomedical Engineering Department of Northwestern University, Evanston, IL. His research interests are time-domain methods in numerical electromagnetics, numerical modeling of electromagnetic wave propagation in multilayered media, numerical electromagnetic simulation of optical systems and numerical simulation of the scattering of light from inhomogeneous/random media.

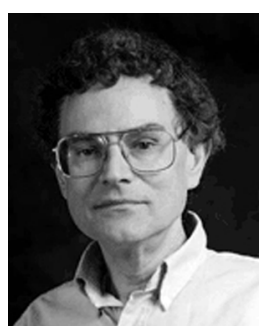

Allen Taflove (M'75-SM'84-F'90) is a Professor of electrical engineering and computer science at Northwestern University, Evanston, IL. Since 1972, he has developed fundamental theoretical approaches, algorithms, and applications of finite-difference time-domain (FDTD) computational solutions of Maxwell's equations. He coined the descriptors "finite difference time domain" and "FDTD" in a 1980 IEEE paper, and in 1990 was the first person to be named an IEEE Fellow in the FDTD technical area. In 2002, he was named by the Institute of Scientific Information (ISI) to its original listing of the most-cited researchers worldwide, as published in ISIHighlyCited.com ${ }^{\circledR}$. To date, his 133 journal papers and four FDTD books have received a total of more than 12,000

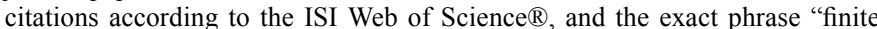
difference time domain" has appeared in over 44,000 articles according to Google Scholar ${ }^{\circledR}$. In May 2010, Nature Milestones: Photons recognized Prof. Taflove as one of the two principal pioneers of numerical methods for solving Maxwell's equations.

Vadim Backman is a Professor of biomedical engineering and the Program Leader of Cancer and Physical Sciences at the Robert H. Lurie Comprehensive Cancer Center of Northwestern University, Evanston, IL. Dr. Backman has 136 peer-reviewed publications and book chapters and 16 patents on biological optical imaging. He is an editor of Biomedical Applications of Light Scattering (McGraw Hill, 2009) and has been a chair of the Conference on Biomedical Applications of Light Scattering since 2007. He has developed and taught classes on fundamentals of microscopy and advanced physical and applied optics. 